\title{
2-LEVEL FRACTIONAL FACTORIAL DESIGNS WHICH ARE THE UNION OF NON TRIVIAL REGULAR DESIGNS
}

\author{
ROBERTO FONTANA AND GIOVANNI PISTONE
}

\begin{abstract}
Every fraction is a union of points, which are trivial regular fractions. To characterize non trivial decomposition, we derive a condition for the inclusion of a regular fraction as follows. Let $F=$ $\sum_{\alpha} b_{\alpha} X^{\alpha}$ be the indicator polynomial of a generic fraction, see Fontana et al, JSPI 2000, 149-172. Regular fractions are characterized by $R=$ $\frac{1}{l} \sum_{\alpha \in \mathcal{L}} e_{\alpha} X^{\alpha}$, where $\alpha \mapsto e_{\alpha}$ is an group homeomorphism from $\mathcal{L} \subset \mathbb{Z}_{2}^{d}$ into $\{-1,+1\}$. The regular $R$ is a subset of the fraction $F$ if $F R=$ $R$, which in turn is equivalent to $\sum_{t} F(t) R(t)=\sum_{t} R(t)$. If $\mathcal{H}=$ $\left\{\alpha_{1} \ldots \alpha_{k}\right\}$ is a generating set of $\mathcal{L}$, and $R=\frac{1}{2^{k}}\left(1+e_{1} X^{\alpha_{1}}\right) \cdots\left(1+e_{k} X^{\alpha_{k}}\right)$, $e_{j}= \pm 1, j=1 \ldots k$, the inclusion condition in term of the $b_{\alpha}$ 's is

$$
b_{0}+e_{1} b_{\alpha_{1}}+\cdots+e_{1} \cdots e_{k} b_{\alpha_{1}+\cdots+\alpha_{k}}=1 .
$$
\end{abstract}

The last part of the paper will discuss some examples to investigate the practical applicability of the previous condition $(*)$.

This paper is an offspring of the Alcotra 158 EU research contract on the planning of sequential designs for sample surveys in tourism statistics.

\section{INTRODUCTION}

We consider 2-level fractional designs with $m$ factors, where the levels of each factor are coded $-1,+1$. The full factorial design is $\mathcal{D}=\{-1,+1\}^{m}$ and a fraction of the full design is a subset $\mathcal{F} \subset \mathcal{D}$. According to the algebraic description of designs, as it is discussed in [7, 6], the fraction ideal Ideal $(\mathcal{F})$, also called design ideal, is the set of all polynomials with real coefficients that are zero on all points of the fraction. Two polynomials $f$ and $g$ are aliased by $\mathcal{F}$ if and only if $f-g \in \operatorname{Ideal}(\mathcal{F})$ and the quotient space defined in such a way is the vector space of real responses on $\mathcal{F}$. The fraction ideal is generated by a finite number of its elements. This finite set of polynomials is called a basis of the ideal. bases are not uniquely determined, unless very special conditions are met. A Gröbner basis of the fraction ideal can be defined after the assignment of a total order on monomials called monomial order. If a monomial order is given, it is possible to identify the leading monomial of each polynomial. As far as applications to statistics are concerned, a Gröbner basis is characterized by the following property: the set of all monomials that are are not divided by any of the leading term of the

Date: Presented by R. Fontana at the DAE 2007 Conference, The University of Memphis, November 2, 2007. 
polynomials in the basis form a linear basis of the quotient vector space. A general reference to the relevant computational commutative algebra topics is $[2]$.

The ring of polynomials in $m$ indeterminates $x_{1} \ldots x_{m}$ and rational coefficient is denoted by $R=\mathbb{Q}\left[x_{1} \ldots x_{m}\right]$. The design ideal Ideal $(\mathcal{D})$ has a unique 'minimal' basis $x_{1}^{2}-1, \ldots, x_{m}^{2}-1$, which happens to be a Gröbner basis. The polynomials that are added to this basis to generate the ideal of a fraction are called generating equations. An ideal with a basis of binomials with coefficients \pm 1 is called binomial ideal. Indicator polynomials polynomials of a fraction were introduced in [3], see also [9]. An indicator polynomial has the form

$$
F=\sum_{\alpha} b_{\alpha} x^{\alpha}, \quad \alpha=\left(\alpha_{1}, \ldots, \alpha_{m}\right) \in\{0,1\}, \quad x^{\alpha}=x_{1}^{\alpha_{1}} \cdots x_{d}^{\alpha_{d}}
$$

and it satisfies the conditions $F(a)=1$ if $a \in \mathcal{F}, F(a)=0$ otherwise. If necessary, we distinguish between the indeterminate $x_{j}$, the value $a_{j}$ and the mapping $X_{j}(a)=a_{j}$. How to move between the ideal representation and the indicator function representation, is discussed in [5].

The definition and characterization, from the algebraic point of view, of regular fractional factorial designs (briefly regular designs) is discussed in [3], see also 9. In particular, the last paper referred to considers mixed factorial design, but this case is outside the scope of the present paper. Orthogonal arrays as are defined in [4] can be characterized in the previous algebraic framework, see [9] and [1], as follows. A fraction $\mathcal{F}$ with indicator polynomial $F$ is orthogonal with strength $s$ if $b_{\alpha}=0$ if $1 \leq|\alpha| \leq s,|\alpha|=\sum_{j} \alpha_{j}$. The notion of indicator polynomial can be accommodated to cases with replicated design points by allowing integer values other than 0 and 1 to $F$, see [11. In such a case, we prefer to call $F$ a counting polynomial of the fraction. A systematic algebraic search of orthogonal arrays with replications is discussed in [1. For sake of easy reference in Section 5 below, we quote a couple of specific result about orthogonal arrays. In fact, considering $m=5$ factors and strength $s=2$, it is shown in [1, Table 5.2] that there are 192 OA's with 12 points and no replications, and there are 32 OA's with 12 points, one of them replicated.

This paper is organized as follows. In Section 2 the algebraic theory is reviewed and in Section 3 it is applied to the problem of finding fractions that are union of regular fractions. In Section 5 the important case of Plackett-Burman designs is considered.

\section{Regular FRACTIONS}

According to the definitions in [3] and [8] a regular fraction is defined as follows. Let $\mathcal{L}$ be a subset of $L=\mathbb{Z}_{2}^{m}$, which is an additive group. Let $\Omega_{2}$ be the multiplicative group $\{-1,+1\}$

Definition 2.1. Let e be a map from $\mathcal{L}$ to $\Omega_{2}$. A non-empty fraction $\mathcal{F}$ is regular if 
(1) $\mathcal{L} \subset L$ s a sub-group;

(2) the equations

$$
X^{\alpha}=e(\alpha) \quad, \quad \alpha \in \mathcal{L}
$$

define the fraction $\mathcal{F}$, i.e. are a set of generating equations.

In such a case, e is a group homeomorphism.

Other known definitions are shown to be equivalent to this one by the following proposition.

Theorem 2.1. Let $\mathcal{F}$ be a fraction. The following statements are equivalent:

(1) The fraction $\mathcal{F}$ is regular according to definition 2.1.

(2) The indicator function of the fraction has the form

$$
F(\zeta)=\frac{1}{l} \sum_{\alpha \in \mathcal{L}} e(\alpha) X^{\alpha}(\zeta), \quad \zeta \in \mathcal{D} .
$$

where $\mathcal{L}$ is a given subset of $L$ and $e: \mathcal{L} \rightarrow \Omega_{2}$ is a given mapping.

(3) For each $\alpha, \beta \in L$ the interactions represented on $\mathcal{F}$ by the terms $X^{\alpha}$ and $X^{\beta}$ are either orthogonal or totally aliased.

(4) The Ideal $(\mathcal{F})$ is binomial.

(5) $\mathcal{F}$ is either a subgroup or a lateral of a subgroup of the multiplicative group $\mathcal{D}$

Proof. Most of the equivalences are either well known or proved in the cited literature. We prove the equivalence of (4) The ideal of a regular design is generated by the basis of the full design and by generating polynomials of the form $X^{\alpha}-e_{\alpha}$, where $e_{\alpha}= \pm 1$; all these polynomials are binomials. Viceversa, if the variety of a binomial ideal is a fraction of $\mathcal{D}$, then all the polynomials $x_{i}^{2}-1$ are contained in its ideal, and every other binomial in the basis, say $x^{\alpha}-e x^{\beta}, e= \pm 1$, is equivalent to the generating polynomial $x^{\alpha+\beta}+e$.

We will show some examples of application of such theorem below. We first will prove two propositions that characterize the simple cases of 1-point and 2-points regular fractions.

Proposition 2.1. Every 1-point fraction is regular

Proof. We can prove the statement using design ideals. A single generic point is $a=\left(a_{1}, \ldots, a_{m}\right) \in \mathcal{D}$. A binomial basis is $\left\{x_{i}-a_{i}, i=1, \ldots, m\right\}$ and, therefore, $\mathcal{F} \equiv\{a\}$ is regular.

Equivalently we can use indicator functions. Indeed the indicator function of a single point $a$ is $F_{a}=\frac{1}{2^{m}}\left(1+a_{1} x_{1}\right) \cdots\left(1+a_{m} x_{m}\right)$ and $F_{a}$ meets the requirements for being an indicator function of a regular design.

The following result looks less trivial.

Proposition 2.2. Every 2-points fraction is regular. 
Proof. Let $\underline{1}=(1, \cdots, 1)$ be the null element of $\mathcal{D}$. We observe that every subset $\mathcal{F}$ of $\mathcal{D}$ made up of two elements, say $a$ and $b$ with $a \neq b$ is a subgroup or a coset of a subgroup. Indeed if $a=\underline{1}$ or $b=\underline{1}$ then $\mathcal{F}$ is a subgroup. If $a \neq \underline{1}$ and $b \neq \underline{1}$ then $\mathcal{F}$ is the coset $a H$ where $H$ is the subgroup $\left\{\underline{1}, a^{-1} b\right\}$.

2.1. Remark. We can also prove the result comparing the number of 2points subsets with the number of subgroups of order 2 . The number of 2 -points fractions of $\mathcal{D}$ is

$$
\left(\begin{array}{c}
2^{m} \\
2
\end{array}\right)=\frac{2^{m} \cdot\left(2^{m}-1\right)}{2}=2^{m-1} \cdot\left(2^{m}-1\right)
$$

On the other side, every regular fraction is a subgroup of $\mathcal{D}$ or a coset of a subgroup of $\mathcal{D}([3])$. In particular the number of regular fractions of size 2 is equivalent to the number of subgroups of order 2 multiplied by the number of cosets of a subgroup, that is $2^{m-1}$.

The number of subgroups of order equal to 2 is $2^{m}-1$. Indeed every set $\{\underline{1}, p\}$ with $\underline{1}=(1, \cdots, 1)$ and $p \in \mathcal{D}, p \neq \underline{1}$ is a subgroup of order equal to 2 .

It follows that the number of regular fractions of size 2 will be equal to

$$
2^{m-1} \cdot\left(2^{m}-1\right)
$$

that is the number of 2-points fraction.

If we consider $2^{k}$-points fractions $(k \geq 2)$ a similar argument is not valid as will be clear in the next sections.

It also follows that every 3-points fraction can be considered as the union of a 1-point fraction and a 2-points fraction.

\section{UNION OF REGULAR DESIGNS}

In this section we consider the union of regular designs. To simplify formulæ we will introduce the following notation:

$$
X^{\alpha} \equiv X_{1}^{\alpha_{1}} \cdots X_{m}^{\alpha_{m}}=X_{\bar{\alpha}}
$$

where $\bar{\alpha}$ is the set for which $\alpha_{i} \neq 0,\left\{i \in\{1, \ldots, m\}: \alpha_{i} \neq 0\right\}$. We will also write $\alpha$ in place of $\bar{\alpha}$ with a small abuse of notation. As an example let's consider $m=4$ and $\alpha=(0,1,1,0)$. It follows that $X^{\alpha}=X_{2} X_{3}$ will be written as $X_{23}$.

Let $\mathcal{F}_{1}$ and $\mathcal{F}_{2}$ two regular fractions, both included in $\mathcal{D}$. The indicator functions of $\mathcal{F}_{1}$ and $\mathcal{F}_{2}$, say $F_{1}$ and $F_{2}$ respectively, allow to easily determine the indicator function of the union of $\mathcal{F}_{1}$ and $\mathcal{F}_{2}, \mathcal{F}=\mathcal{F}_{1} \cup \mathcal{F}_{2}$ as

$$
F=F_{1}+F_{2}-F_{1} \times F_{2}
$$

In general, the union of two (disjoint) regular fractions is not a regular fraction. As an example let's consider $m=2$ factors, $\mathcal{D}=\{-1,+1\} \times$ $\{-1,+1\}$ and $\mathcal{F}_{1}=\{(-1,-1)\}$ and $\left.\mathcal{F}_{2}=\{(-1,+1),(+1,-1))\right\}$. Both $\mathcal{F}_{1}$ 
and $\mathcal{F}_{2}$ are regular fractions, according to the propositions of the previous sections. Indeed their indicator functions meet the requirements for regular fractions: $F_{1}=\frac{1}{4}\left(1-X_{1}\right) \cdot\left(1-X_{2}\right)$ and $F_{2}=\frac{1}{2}\left(1-X_{1} \cdot X_{2}\right)$. However, the union $\mathcal{F}=\{(-1,-1),(-1,+1),(+1,-1))\}$, is not a regular fraction, because its indicator function is

$$
F=\frac{3}{4}-\frac{1}{4} X_{1}-\frac{1}{4} X_{2}-\frac{1}{4} X_{1} \cdot X_{2} .
$$

The same conclusion can be obtained considering design ideals related to fractional designs. Given $\mathcal{F}_{1} \subset \mathcal{D}, \mathcal{F}_{2} \subset \mathcal{D}$ and $\mathcal{F}=\mathcal{F}_{1} \cup \mathcal{F}_{2}$ the associated ideals will be Ideal $\left(\mathcal{F}_{1}\right)$, Ideal $\left(\mathcal{F}_{2}\right)$ and Ideal $(\mathcal{F})$. In general, the fact that Ideal $\left(\mathcal{F}_{1}\right)$ and Ideal $\left(\mathcal{F}_{2}\right)$ are binomial ideals by Theorem ?? doesn't imply that Ideal $(\mathcal{F}))$ is a binomial ideal . Indeed, for the previous example, the Gröbner bases $B_{1}, B_{2}$ and $B$ of $\operatorname{Ideal}\left(\mathcal{F}_{1}\right)$, Ideal $\left(\mathcal{F}_{2}\right)$ and $\operatorname{Ideal}(\mathcal{F})$ respectively, are:

$$
\begin{aligned}
B_{1} & =\left\{X_{1}+1, X_{2}+1\right\} \\
B_{2} & =\left\{X_{2}^{2}-1, X_{1}+X_{2}\right\} \\
B & =\left\{-1 / 4 X_{1} X_{2}-1 / 4 X_{1}-1 / 4 X_{2}-1 / 4, X_{2}^{2}-1, X_{1}^{2}-1\right\}
\end{aligned}
$$

It results that $\operatorname{Ideal}\left(\mathcal{F}_{1}\right)$ and $\operatorname{Ideal}\left(\mathcal{F}_{2}\right)$ are binomial ideals while $\operatorname{Ideal}(\mathcal{F})$ is not.

3.1. Remark. More generally, let's consider two disjoint regular fractions, namely $a G$ and $b H$, where $G$ and $H$ are subgroups of $\mathcal{D}$ and $a \notin G$ and $b \notin H$. Let's take $a g$ and $b h$. In order to have $(a g)(b h) \in a G$ we should have $b g h \in G$ or, equivalently, $b h \in G$.

\section{Decomposing a Fraction into Regular fractions}

In this part of the work we would like to explore the inverse path, i.e. to analyze the decomposition of a given $\mathcal{F} \subset \mathcal{D}$ into the union of disjoint regular fractions. We will indicate with $\mathcal{R}$ the generic regular fraction.

Let's indicate with $F$ and $R$ the indicator functions of $\mathcal{F} \subset \mathcal{D}$ and $\mathcal{R} \subset \mathcal{D}$ respectively. Under which condition $\mathcal{R}$ will be a subset of $\mathcal{F}$ ?

Theorem 4.1. Let $F$ be the indicator function of a generic fractional design $\mathcal{F} \subset \mathcal{D}, F=\sum_{\alpha} b_{\alpha} X^{\alpha}$. Let $R$ the indicator function of a regular fractional design $\mathcal{R} \subset \mathcal{D}, R=\frac{1}{l} \sum_{\alpha \in \mathcal{L}} e_{\alpha} X^{\alpha}=\frac{1}{2^{k}}\left(1+e_{1} X^{\alpha_{1}}\right) \cdots\left(1+e_{k} X^{\alpha_{k}}\right)$. The following statement holds:

$$
\mathcal{R} \subseteq \mathcal{F} \Leftrightarrow b_{0}+e_{1} b_{\alpha_{1}}+\cdots+e_{1} \cdots e_{k} b_{\alpha_{1}+\cdots+\alpha_{k}}=1
$$

Proof. For $\mathcal{R}$ to be a subset of $\mathcal{F}$ it must happen that the number of points of $\mathcal{R}$ must be equal to the number of points of $\mathcal{R} \cap \mathcal{F}$. In terms of indicator functions the equality $\mathcal{R}=\mathcal{R} \cap \mathcal{F}$ becomes $\sum_{t} F(t) R(t)=\sum_{t} R(t)$ being $t \in \mathcal{D}$. We have 


$$
\begin{gathered}
F R= \\
\left(\sum_{\alpha} b_{\alpha} X^{\alpha}\right) \cdot \frac{1}{2^{k}}\left(1+e_{1} X^{\alpha_{1}}\right) \cdots\left(1+e_{k} X^{\alpha_{k}}\right)= \\
\frac{1}{2^{k}} \sum_{\alpha} b_{\alpha} X^{\alpha}+\frac{1}{2^{k}} \sum_{\alpha} b_{\alpha} X^{\alpha} e_{1} X^{\alpha_{1}} \cdots+\frac{1}{2^{k}} \sum_{\alpha} b_{\alpha} X^{\alpha} e_{1} \cdots e_{k} X^{\alpha_{1}+\cdots+\alpha_{k}}
\end{gathered}
$$

It follows that

$$
\sum_{t} F(t) R(t)=\frac{1}{2^{k}} 2^{m} b_{0}+\frac{1}{2^{k}} 2^{m} e_{1} b_{\alpha_{1}}+\cdots \frac{1}{2^{k}} 2^{m} e_{1} \cdots e_{k} b_{\alpha_{1}+\cdots+\alpha_{k}}
$$

On the other hand

$$
\sum_{t} R(t)=\frac{1}{2^{k}} 2^{m}
$$

It follows

$$
b_{0}+e_{1} b_{\alpha_{1}}+\cdots+e_{1} \cdots e_{k} b_{\alpha_{1}+\cdots+\alpha_{k}}=1
$$

Corollary 4.1.1. A necessary, but not sufficient, condition for a regular fraction $\mathcal{R}$ to be contained in $\mathcal{D}$ is

$$
b_{0}+\left|b_{\alpha_{1}}\right|+\cdots+\left|b_{\alpha_{1}+\cdots+\alpha_{k}}\right| \geq 1
$$

4.1. A small example. Let's consider the 3-points fraction $\mathcal{F} \subset \mathcal{D}=$ $\{-1,+1\} \times\{-1,+1\}$ that we have introduced in the previous section:

$$
\mathcal{F}=\{(-1,-1),(-1,+1),(+1,-1))\}
$$

The indicator function $F$ of $\mathcal{F}$ is $F=\frac{3}{4}-\frac{1}{4} X_{1}-\frac{1}{4} X_{2}-\frac{1}{4} X_{1} \cdot X_{2}$, that is $b_{0}=\frac{3}{4}, b_{1}=-\frac{1}{4}, b_{2}=-\frac{1}{4}, b_{12}=-\frac{1}{4}$. It follows

$$
\begin{aligned}
b_{0}-b_{1} & =1 \\
b_{0}-b_{2} & =1 \\
b_{0}-b_{12} & =1 \\
b_{0}-b_{1}-b_{2}+b_{12} & =1 \\
b_{0}-b_{1}+b_{2}-b_{12} & =1 \\
b_{0}+b_{1}-b_{2}-b_{12} & =1
\end{aligned}
$$


From each relation, using theorem 4.1, we can obtain the indicator functions of the regular fractions that are contained into $\mathcal{F}$. These are

$$
\begin{array}{r}
F_{1}=\frac{1}{2}\left(1-X_{1}\right) \\
F_{2}=\frac{1}{2}\left(1-X_{2}\right) \\
F_{3}=\frac{1}{2}\left(1-X_{1} \cdot X_{2}\right) \\
F_{4}=\frac{1}{4}\left(1-X_{1}\right) \cdot\left(1-X_{2}\right) \\
F_{5}=\frac{1}{4}\left(1-X_{1}\right) \cdot\left(1+X_{2}\right) \\
F_{6}=\frac{1}{4}\left(1+X_{1}\right) \cdot\left(1-X_{2}\right)
\end{array}
$$

Therefore the corresponding regular fractions are, respectively

$$
\begin{array}{r}
\left.\mathcal{F}_{1}=\{(-1,-1),(-1,+1))\right\} \\
\left.\mathcal{F}_{2}=\{(-1,-1),(+1,-1))\right\} \\
\left.\mathcal{F}_{3}=\{(-1,+1),(+1,-1))\right\} \\
\mathcal{F}_{4}=\{(-1,-1)\} \\
\mathcal{F}_{5}=\{(-1,+1)\} \\
\mathcal{F}_{6}=\{(+1,-1)\}
\end{array}
$$

\section{Plackett-Burman Designs}

Another example can be obtained considering the well-known "PlackettBurman" designs [10]. In particular the Plackett-Burman design for 11 variables and 12 runs is built according the following procedure:

(1) the first row, namely the key, is given: ++-+++---+-

(2) the second row up to the eleventh row are built shifting the key of one position each time

(3) the 12 th row is set equal to -----------

The Plackett-Burman design for eleven parameters becomes 


$$
\begin{array}{cccccccccccc}
N & A & B & C & D & E & F & G & H & I & J & K \\
1 & + & + & - & + & + & + & - & - & - & + & - \\
2 & - & + & + & - & + & + & + & - & - & - & + \\
3 & + & - & + & + & - & + & + & + & - & - & - \\
4 & - & + & - & + & + & - & + & + & + & - & - \\
5 & - & - & + & - & + & + & - & + & + & + & - \\
6 & - & - & - & + & - & + & + & - & + & + & + \\
7 & + & - & - & - & + & - & + & + & - & + & + \\
8 & + & + & - & - & - & + & - & + & + & - & + \\
9 & + & + & + & - & - & - & + & - & + & + & - \\
10 & - & + & + & + & - & - & - & + & - & + & + \\
11 & + & - & + & + & + & - & - & - & + & - & + \\
12 & - & - & - & - & - & - & - & - & - & - & -
\end{array}
$$

We consider the case with $m=5$ factors and, from the "Plackett-Burman" for 11 factors we randomly select the following $\mathcal{F}$, corresponding to columns $\mathrm{A}, \mathrm{B}, \mathrm{F}, \mathrm{H}$ and I of the original design.

The plus sign ' + ' has been coded with ' 1 ' and the minus sign '-' with '-1'.

$$
\mathcal{F}=\begin{array}{rrrrrr}
N & X_{1} & X_{2} & X_{3} & X_{4} & X_{5} \\
1 & 1 & 1 & 1 & 1 & 1 \\
2 & 1 & 1 & -1 & -1 & 1 \\
3 & 1 & -1 & -1 & -1 & 1 \\
4 & -1 & 1 & -1 & 1 & 1 \\
5 & -1 & -1 & 1 & 1 & 1 \\
6 & -1 & -1 & 1 & -1 & 1 \\
7 & 1 & 1 & 1 & -1 & -1 \\
8 & 1 & -1 & 1 & 1 & -1 \\
9 & 1 & -1 & -1 & 1 & -1 \\
10 & -1 & 1 & 1 & -1 & -1 \\
11 & -1 & 1 & -1 & 1 & -1 \\
12 & -1 & -1 & -1 & -1 & -1
\end{array}
$$

The indicator function $F$ of $\mathcal{F}$ is

$$
\begin{aligned}
\frac{3}{8}+\frac{1}{8} X_{345}+\frac{1}{8} X_{245}- & \frac{1}{8} X_{235}-\frac{1}{8} X_{234}+ \\
\frac{1}{8} X_{2345}-\frac{1}{8} X_{145} & -\frac{1}{8} X_{135}+\frac{1}{8} X_{134}+\frac{1}{8} X_{1345}+\frac{1}{8} X_{125}+ \\
& -\frac{1}{8} X_{124}+\frac{1}{8} X_{1245}+\frac{1}{8} X_{123}+\frac{1}{8} X_{1235}+\frac{1}{8} X_{1234}
\end{aligned}
$$

It follows that $\mathcal{F}$ is not regular.

Now we start to search for regular fractions that are contained in $\mathcal{F}$. 
Of course the first constraint concerns the size of the regular fraction. It must be less or equal to 12 , the number of points of $\mathcal{F}$. Being $\mathcal{R}$ a regular fraction, it follows that the size of $\mathcal{R}$ could be $2^{0}=1$ or $2^{1}=2$ or $2^{2}=4$ or $2^{3}=8$.

We already know, from the propositions of section 2 that

- all the 12 points that constitute $\mathcal{R}$ are 1-point regular fraction;

- all the $\left(\begin{array}{c}12 \\ 2\end{array}\right)=66$ are 2 -points regular fraction.

Let's study 4-points and 8-points subsets of $\mathcal{F}$.

The corollary of theorem Th. 4.1 allows us to immediately exclude 8points regular fractions. Indeed the following condition should be true for a proper choice of $e_{1}, e_{2}$ and $\alpha_{1}, \alpha_{2}$

$$
b_{0}+e_{1} b_{\alpha_{1}}+e_{2} b_{\alpha_{2}}+e_{1} e_{2} b_{\alpha_{1}+\alpha_{2}}=1
$$

But $b_{0}=\frac{3}{8}$ and the absolute value of $b_{i}$ is $\frac{1}{8}, \forall i$ and so it is not possible that the left side of the previous equation sums up to 1 . No 8-points regular fraction is contained into $\mathcal{F}$.

Finally we investigate 4 -points regular fractions.

For a proper choice of $e_{1}, e_{2}, e_{3}$ and $\alpha_{1}, \alpha_{2}, \alpha_{3}$ the following relation should hold

$b_{0}+e_{1} b_{\alpha_{1}}+e_{2} b_{\alpha_{2}}+e_{3} b_{\alpha_{3}}+e_{1} e_{2} b_{\alpha_{1}+\alpha_{2}}+e_{1} e_{3} b_{\alpha_{1}+\alpha_{3}}+e_{2} e_{3} b_{\alpha_{2}+\alpha_{3}}+e_{1} e_{2} e_{3} b_{\alpha_{1}+\alpha_{2}+\alpha_{3}}=1$

A subgroup of order eight will be $\{\underline{1}, a, b, a b, c, a c, b c, a b c\}$ with $a \neq \underline{1}$, $b \neq \underline{1}, c \neq 1$ and $a \neq b, a \neq c$ and $b \neq c$. We can choose $a, b$ and $c$ in $\left(\begin{array}{c}31 \\ 2\end{array}\right) \cdot(31-3)$ different ways. The number of different subgroups is obtained dividing this number by $\left(\begin{array}{l}7 \\ 2\end{array}\right) \cdot 4$. We get 155 different subgroups.

Every subgroup of order $8, \mathcal{S}_{i}^{(8)}=<\alpha_{1 i}, \alpha_{2 i}, \alpha_{3 i}>, i=1, \ldots, 155$ defines 8 regular fractions of size 4 (the subgroup orthogonal to $\mathcal{S}_{i}^{(8)}$ and its cosets). To find the regular fractions embedded into $\mathcal{F}$ we must solve the following systems of equations $(i=1, \ldots, 155)$

$$
\left\{\begin{array}{l}
e_{1}^{2}-1=0 \\
e_{2}^{2}-1=0 \\
e_{3}^{2}-1=0 \\
b_{0}+e_{1} b_{\alpha_{1 i}}+e_{2} b_{\alpha_{2 i}}+e_{3} b_{\alpha_{3 i}}+e_{1} e_{2} b_{\alpha_{1 i}+\alpha_{2 i}}+e_{1} e_{3} b_{\alpha_{1 i}+\alpha_{3 i}}+e_{2} e_{3} b_{\alpha_{2 i}+\alpha_{3 i}}+e_{1} e_{2} e_{3} b_{\alpha_{1 i}+\alpha_{2 i}+\alpha_{3 i}}-1=0
\end{array}\right.
$$

To do it we generate the 155 subgroups of $\mathcal{D}$ of order eight (for example using the package GAP [?]). As an example let's consider $\mathcal{S}_{1}=<\{1\},\{2\},\{3\}>$. Being $b_{0}=\frac{3}{8}, b_{1}=b_{2}=b_{3}=b_{12}=b_{13}=b_{23}=0$ and $b_{123}=\frac{1}{8}$ the 
corresponding system of equation is

$$
\left\{\begin{array}{l}
e_{1}^{2}-1=0 \\
e_{2}^{2}-1=0 \\
e_{3}^{2}-1=0 \\
\frac{3}{8}+\frac{1}{8} e_{1} e_{2} e_{3}-1=0
\end{array}\right.
$$

The system doesn't have any solution.

Let's now consider $\mathcal{S}_{2}=<\{4\},\{12\},\{135\}>$. Being $b_{0}=\frac{3}{8}, b_{4}=b_{1} 2=$ $0, b_{135}=b_{124}=b_{235}=-\frac{1}{8}$ and $b_{1345}=b_{2345}=\frac{1}{8}$ the corresponding system of equation is

$$
\left\{\begin{array}{l}
e_{1}^{2}-1=0 \\
e_{2}^{2}-1=0 \\
e_{3}^{2}-1=0 \\
\frac{3}{8}-\frac{1}{8} e_{3}-\frac{1}{8} e_{1} e_{2}+\frac{1}{8} e_{1} e_{3}-\frac{1}{8} e_{2} e_{3}+\frac{1}{8} e_{1} e_{2} e_{3}-1=0
\end{array}\right.
$$

The system has the following solution $e_{1}=-1, e_{2}=1, e_{3}=-1$ that defines the following indicator function $F^{(1)}$

$$
\frac{1}{8}\left(1-X_{4}\right)\left(1+X_{12}\right)\left(1-X_{135}\right)
$$

The corresponding set of points $\mathcal{F}^{(1)}$ is

$$
\begin{array}{crrrrr}
N & X_{1} & X_{2} & X_{3} & X_{4} & X_{5} \\
2 & 1 & 1 & -1 & -1 & 1 \\
6 & -1 & -1 & 1 & -1 & 1 \\
7 & 1 & 1 & 1 & -1 & -1 \\
12 & -1 & -1 & -1 & -1 & -1
\end{array}
$$

To proceed into the decomposition of $\mathcal{F}$ we remove the points of $\mathcal{F}^{(1)}$. The indicator function of the new set will be $F-F^{(1)}$ :

$$
\begin{aligned}
\frac{1}{4}+\frac{1}{8} X_{4}- & \frac{1}{8} X_{12}+\frac{1}{8} X_{345}+\frac{1}{8} X_{245}-\frac{1}{8} X_{234}+ \\
-\frac{1}{8} X_{145}+\frac{1}{8} X_{134}+\frac{1}{8} X_{125}+\frac{1}{8} X_{1245}+ & \frac{1}{8} X_{123}+ \\
& +\frac{1}{8} X_{1235}+\frac{1}{8} X_{1234}
\end{aligned}
$$

We now search for the regular fractions contained into $\mathcal{F}-\mathcal{F}^{(1)}$. A regular fraction $\mathcal{R}$ to be contained into $\mathcal{F}-\mathcal{F}^{(1)}$ must be contained into $\mathcal{F}$. We can therefore limit our search to the solution that we have identified in the first part. Let's now consider $\mathcal{S}_{3}=<\{12\},\{35\},\{245\}>$. 
Being $b_{0}^{(1)}=\frac{1}{4}, b_{35}^{(1)}=0 b_{245}^{(1)}=b_{134}^{(1)}=b_{1235}^{(1)}=\frac{1}{8}$ and $b_{234}^{(1)}=b_{145}^{(1)}=b_{12}^{(1)}=$ $-\frac{1}{8}$ the corresponding system of equation is

$$
\left\{\begin{array}{l}
e_{1}^{2}-1=0 \\
e_{2}^{2}-1=0 \\
e_{3}^{2}-1=0 \\
\frac{1}{4}-\frac{1}{8} e_{1}+\frac{1}{8} e_{3}+\frac{1}{8} e_{1} e_{2}-\frac{1}{8} e_{1} e_{3}-\frac{1}{8} e_{2} e_{3}+\frac{1}{8} e_{1} e_{2} e_{3}-1=0
\end{array}\right.
$$

The system has the following solution $e_{1}=-1, e_{2}=-1, e_{3}=1$ that defines the following indicator function $F^{(2)}$

$$
\frac{1}{8}\left(1-X_{12}\right)\left(1-X_{35}\right)\left(1+X_{245}\right)
$$

The corresponding set of points $\mathcal{F}^{(2)}$ is

$$
\begin{array}{crrrrr}
N & X_{1} & X_{2} & X_{3} & X_{4} & X_{5} \\
3 & 1 & -1 & -1 & -1 & 1 \\
4 & -1 & 1 & -1 & 1 & 1 \\
8 & 1 & -1 & 1 & 1 & -1 \\
10 & -1 & 1 & 1 & -1 & -1
\end{array}
$$

If we remove this set of points from $\mathcal{F}-\mathcal{F}_{1}$ we get the following indicator function $F^{(3)}=F-F^{(1)}-F^{(2)}$ :

$$
\frac{1}{8}+\frac{1}{8} X_{4}+\frac{1}{8} X_{35}+\frac{1}{8} X_{345}+\frac{1}{8} X_{125}+\frac{1}{8} X_{1245}+\frac{1}{8} X_{123}+\frac{1}{8} X_{1234}
$$

or, equivalently,

$$
\frac{1}{8}\left(1+X_{4}\right)\left(1+X_{35}\right)\left(1+X_{125}\right)
$$

and the corresponding set of points $\mathcal{F}^{(3)}$

$\begin{array}{crrrrr}N & X_{1} & X_{2} & X_{3} & X_{4} & X_{5} \\ 1 & 1 & 1 & 1 & 1 & 1 \\ 5 & -1 & -1 & 1 & 1 & 1 \\ 9 & 1 & -1 & -1 & 1 & -1 \\ 11 & -1 & 1 & -1 & 1 & -1\end{array}$

$F^{(3)}$ meets the requirements to be an indicator function of a regular design. We have therefore decomposed $\mathcal{F}$ into three regular designs, $\mathcal{F}=$ $\mathcal{F}_{1} \cup \mathcal{F}_{2} \cup \mathcal{F}_{3}$.

\subsection{Decomposition of the given 'Plackett-Burman' design into all} the unions of 4-points regular designs. In this part we find all the possible decompositions of the given "Plackett-Burman" design. As described in the previous section, we consider all the 155 subgroups of order $8, \mathcal{S}_{i}^{(8)}=<\alpha_{1 i}, \alpha_{2 i}, \alpha_{3 i}>, i=1, \ldots, 155$ and we search for the solution of the following systems of equations 
$\left\{\begin{array}{l}e_{1}^{2}-1=0 \\ e_{2}^{2}-1=0 \\ e_{3}^{2}-1=0 \\ b_{0}+e_{1} b_{\alpha_{1 i}}+e_{2} b_{\alpha_{2 i}}+e_{3} b_{\alpha_{3 i}}+e_{1} e_{2} b_{\alpha_{1 i}+\alpha_{2 i}}+e_{1} e_{3} b_{\alpha_{1 i}+\alpha_{3 i}}+e_{2} e_{3} b_{\alpha_{2 i}+\alpha_{3 i}}+e_{1} e_{2} e_{3} b_{\alpha_{1 i}+\alpha_{2 i}+\alpha_{3 i}}-1=0\end{array}\right.$

15 of these 155 systems of equations have a non-empty set of solutions. Each of these non-empty sets define an indicator function $R_{j}, j=1, \cdots, 15$ :

$$
\begin{aligned}
& R_{1}=\frac{1}{8}\left(1-X_{4}\right)\left(1+X_{12}\right)\left(1-X_{235}\right) \\
& R_{2}=\frac{1}{8}\left(1+X_{1}\right)\left(1+X_{23}\right)\left(1+X_{245}\right) \\
& R_{3}=\frac{1}{8}\left(1+X_{1}\right)\left(1-X_{45}\right)\left(1-X_{235}\right) \\
& R_{4}=\frac{1}{8}\left(1-X_{2}\right)\left(1+X_{34}\right)\left(1-X_{145}\right) \\
& R_{5}=\frac{1}{8}\left(1+X_{2}\right)\left(1+X_{15}\right)\left(1-X_{345}\right) \\
& R_{6}=\frac{1}{8}\left(1-X_{23}\right)\left(1-X_{45}\right)\left(1-X_{135}\right) \\
& R_{7}=\frac{1}{8}\left(1-X_{3}\right)\left(1+X_{25}\right)\left(1-X_{145}\right) \\
& R_{8}=\frac{1}{8}\left(1+X_{3}\right)\left(1+X_{14}\right)\left(1-X_{245}\right) \\
& R_{9}=\frac{1}{8}\left(1-X_{14}\right)\left(1-X_{25}\right)\left(1+X_{345}\right) \\
& R_{10}=\frac{1}{8}\left(1-X_{15}\right)\left(1-X_{34}\right)\left(1+X_{245}\right) \\
& R_{11}=\frac{1}{8}\left(1-X_{5}\right)\left(1+X_{13}\right)\left(1-X_{234}\right) \\
& R_{12}=\frac{1}{8}\left(1+X_{4}\right)\left(1+X_{35}\right)\left(1-X_{125}\right) \\
& R_{13}=\frac{1}{8}\left(1+X_{5}\right)\left(1+X_{24}\right)\left(1-X_{134}\right) \\
& R_{14}=\frac{1}{8}\left(1-X_{12}\right)\left(1-X_{35}\right)\left(1+X_{245}\right) \\
& R_{15}=\frac{1}{8}\left(1-X_{13}\right)\left(1-X_{24}\right)\left(1+X_{345}\right)
\end{aligned}
$$

To build a generic decomposition of $\mathcal{F}$ we start from one of these indicator function, let's say $R_{1}$ that identify the regular fraction $\mathcal{R}_{1}$. We have now to choose another indicator functions in the set made up by $R_{2}, \ldots, R_{15}$, let's say $R_{k}$, with the condition that the corresponding regular fraction $\mathcal{R}_{k}$ doesn't intersect $\mathcal{R}_{1}: \mathcal{R}_{1} \cap \mathcal{R}_{k}=\emptyset$. We have two possible choices, $R_{12}$ and $R_{14}$. If we choose $R_{12}$ the only possible remaining is $R_{14}$ and, viceversa, if we choose $R_{14}$ the only possible remaining is $R_{12}$. Repeating the same procedure for all the $R_{i}$ and considering only the different decompositions, we get that $\mathcal{F}$ can be considered as the union of three regular 4-points designs

$$
\begin{aligned}
& \mathcal{F}=\mathcal{R}_{1} \cup \mathcal{R}_{12} \cup \mathcal{R}_{14} \\
& \mathcal{F}=\mathcal{R}_{2} \cup \mathcal{R}_{3} \cup \mathcal{R}_{6} \\
& \mathcal{F}=\mathcal{R}_{4} \cup \mathcal{R}_{5} \cup \mathcal{R}_{10} \\
& \mathcal{F}=\mathcal{R}_{7} \cup \mathcal{R}_{8} \cup \mathcal{R}_{9} \\
& \mathcal{F}=\mathcal{R}_{11} \cup \mathcal{R}_{13} \cup \mathcal{R}_{15}
\end{aligned}
$$

The decomposition that has been found in the previous section is $\mathcal{F}=$ $\mathcal{R}_{1} \cup \mathcal{R}_{12} \cup \mathcal{R}_{14}$.

5.2. Decomposition of all the "Plackett-Burman" designs with $\mathbf{m}=\mathbf{5}$ and 12 different runs into all the unions of 4-points regular designs. Using an ad-hoc software routine written in SAS IML we consider all 
the $\left(\begin{array}{c}11 \\ 5\end{array}\right)=462$ different designs that can be obtained choosing 5 columns out of the 11 of the original designs. We get the following table where the first column contains an identification of the design, the second column the number of designs that are equal to the design and the third column the number of different runs contained in the design. For example, the design $\mathcal{F}$ that we have considered in the previous sections, belongs to the class "69". There are 11 designs that are equal to $\mathcal{F}$ and each has 12 points.

\begin{tabular}{|rrr|rrr|rrr|}
\multicolumn{1}{rl}{$I D$} & $N$ & $S I Z E$ & \multicolumn{1}{c}{$I D$} & $N$ & $S I Z E$ & $I D$ & $N$ & $S I Z E$ \\
\hline 1 & 8 & 12 & 28 & 6 & 12 & 72 & 5 & 11 \\
2 & 7 & 12 & 30 & 10 & 12 & 73 & 6 & 12 \\
3 & 6 & 12 & 32 & 6 & 11 & 74 & 5 & 12 \\
4 & 8 & 12 & 35 & 6 & 12 & 82 & 6 & 12 \\
5 & 5 & 12 & 37 & 3 & 12 & 84 & 2 & 12 \\
6 & 7 & 11 & 39 & 4 & 12 & 85 & 9 & 11 \\
7 & 2 & 12 & 44 & 11 & 12 & 87 & 7 & 12 \\
8 & 13 & 12 & 45 & 7 & 12 & 89 & 4 & 12 \\
9 & 6 & 12 & 46 & 6 & 12 & 94 & 6 & 12 \\
10 & 11 & 11 & 49 & 2 & 12 & 98 & 7 & 12 \\
11 & 7 & 12 & 51 & 7 & 12 & 100 & 3 & 12 \\
12 & 7 & 12 & 52 & 9 & 12 & 101 & 8 & 12 \\
13 & 5 & 12 & 53 & 5 & 12 & 102 & 3 & 11 \\
14 & 7 & 11 & 54 & 4 & 11 & 103 & 7 & 12 \\
15 & 10 & 12 & 55 & 4 & 12 & 110 & 2 & 12 \\
16 & 6 & 12 & 57 & 3 & 11 & 116 & 5 & 12 \\
17 & 7 & 12 & 58 & 6 & 12 & 117 & 1 & 12 \\
18 & 3 & 12 & 61 & 6 & 12 & 128 & 2 & 12 \\
19 & 7 & 12 & 63 & 4 & 12 & 134 & 5 & 12 \\
20 & 11 & 12 & 64 & 3 & 12 & 140 & 3 & 12 \\
21 & 5 & 12 & 65 & 8 & 12 & 146 & 5 & 11 \\
22 & 8 & 12 & 66 & 5 & 12 & 147 & 3 & 12 \\
23 & 4 & 12 & 67 & 2 & 12 & 149 & 4 & 12 \\
24 & 7 & 12 & 68 & 7 & 12 & 154 & 6 & 12 \\
25 & 2 & 12 & 69 & 11 & 12 & 159 & 1 & 12 \\
26 & 5 & 12 & 70 & 13 & 12 & 167 & 2 & 12 \\
27 & 6 & 11 & 71 & 6 & 12 & 184 & 1 & 12
\end{tabular}

It follows that the 462 designs can be partitioned into 81 classes:

- there are 70 classes where each design contains 12 runs

- there are 11 classes where each design contains 11 runs

We limit to designs with 12 different runs. We repeat the procedure described in the previous section for all the 70 different designs. First of all we determine the indicator functions of all the 70 designs. Every indicator function has the following form:

$$
\begin{aligned}
& \frac{3}{8}+a_{345} X_{345}+a_{245} X_{245}+a_{235} X_{235}+a_{234} X_{234}+ \\
& a_{2345} X_{2345}+a_{145} X_{145}+a_{135} X_{135}+a_{134} X_{134}+a_{1345} X_{1345}+a_{125} X_{125}+ \\
& a_{124} X_{124}+a_{1245} X_{1245}+a_{123} X_{123}+a_{1235} X_{1235}+a_{1234} X_{1234}
\end{aligned}
$$

where the coefficients $a_{345}, \ldots, a_{1234}$ are equal to $\pm \frac{1}{8}$.

We decompose every fraction into three 4-points regular design.

As for the design considered in the previous example we have that every design

- contains 15 "4-points regular design" 
- can be considered as the union of three regular designs in 5 different ways

We have examined the decomposition structure of all the 70 designs. If we indicate with $R_{1}, R_{2}$ and $R_{3}$ the indicator functions of the regular designs contained into one of the design, we get

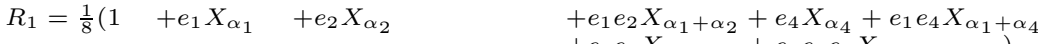

$$
\begin{aligned}
& R_{2}=\frac{1}{8}\left(1-e_{1} X_{\alpha_{1}} \quad+e_{2} X_{2} e_{4} X_{\alpha_{2}+\alpha_{4}}+e_{1} e_{2} e_{4} X_{\alpha_{1}+\alpha_{2}+\alpha_{4}}\right)
\end{aligned}
$$

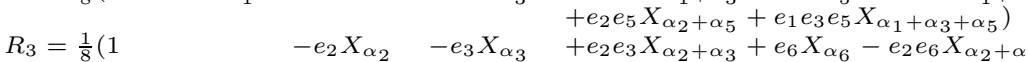

$$
\begin{aligned}
& -e_{3} e_{6} X_{\alpha_{3}+\alpha_{6}}+e_{2} e_{3} e_{6} X_{\alpha_{2}+\alpha_{3}+\alpha_{6}} \text { ) }
\end{aligned}
$$

where, being $|\alpha|=\sum_{j} \alpha_{j}$,

- $\left|\alpha_{1}\right|,\left|\alpha_{2}\right|$ and $\left|\alpha_{3}\right|$ are all less than three

- all the others, i.e. $\left|\alpha_{1}+\alpha_{2}\right|, \ldots,\left|\alpha_{2}+\alpha_{3}+\alpha_{6}\right|$ are all greater or equal to 3

This evidence has suggested the following procedure.

(1) We have built all the $\alpha_{1}, \ldots, \alpha_{6}$ that satisfy the previous requirement,

\begin{tabular}{l|cccccc}
$N$ & $\alpha_{1}$ & $\alpha_{2}$ & $\alpha_{3}$ & $\alpha_{4}$ & $\alpha_{5}$ & $\alpha_{6}$ \\
1 & 1 & 23 & 45 & 245 & 234 & 124 \\
2 & 1 & 24 & 35 & 235 & 234 & 123 \\
3 & 1 & 25 & 34 & 234 & 235 & 123 \\
4 & 2 & 13 & 45 & 145 & 134 & 124 \\
5 & 2 & 14 & 35 & 135 & 134 & 123 \\
6 & 2 & 15 & 34 & 134 & 135 & 123 \\
7 & 3 & 12 & 45 & 145 & 124 & 134 \\
8 & 3 & 14 & 25 & 125 & 124 & 123 \\
9 & 3 & 15 & 24 & 124 & 125 & 123 \\
10 & 4 & 12 & 35 & 135 & 123 & 134 \\
11 & 4 & 13 & 25 & 125 & 123 & 124 \\
12 & 4 & 15 & 23 & 123 & 125 & 124 \\
13 & 5 & 12 & 34 & 134 & 123 & 135 \\
14 & 5 & 13 & 24 & 124 & 123 & 125 \\
15 & 5 & 14 & 23 & 123 & 124 & 125
\end{tabular}

(2) For every choice of $\alpha_{1}, \ldots, \alpha_{6}$ we have built the 64 indicator functions that correspond to all the values of $e_{1}, \ldots, e_{6}$, being $e_{i}= \pm 1, i=$ $1, \ldots, 6$.

According to this procedure we have generated $15 \times 64=960$ indicator functions. If we limit to the different ones we get 192 indicator functions. This number is the same that has been found in [1], as the total number of orthogonal arrays of strength 2 .

5.3. Remark. It is interesting to point out that the "understanding" of the mechanism underlying the Plackett-Burman designs ( $m=5$, 12-runs) has allowed to build all the orthogonal arrays of strength 2 . 


\section{Conclusions}

- The problem to determine regular designs that are contained in a given fraction has been faced.

- A condition in terms of the coefficients of the polynomial indicator function has been found.

- The decomposition of a given fraction into regular designs seems useful for fractional factorial generation.

\section{REFERENCES}

[1] Enrico Carlini and Giovanni Pistone. Hibert bases for orthogonal arrays. Journal of Statistical Theory and practice. Accepted 29-05-2007. Preprint arXiv:math/0611276.

[2] David Cox, John Little, and Donal O'Shea. Ideals, varieties, and algorithms. Undergraduate Texts in Mathematics. Springer-Verlag, New York, second edition, 1997. An introduction to computational algebraic geometry and commutative algebra.

[3] Roberto Fontana, Giovanni Pistone, and Maria Piera Rogantin. Classification of twolevel factorial fractions. Journal of Statistical Planning and Inference, 87(1):149-172, May 2000.

[4] A. S. Hedayat, N. J. A. Sloane, and John Stufken. Orthogonal arrays. Theory and applications. Springer Series in Statistics. Springer-Verlag, New York, 1999.

[5] Roberto Notari, Eva Riccomagno, and Maria-Piera Rogantin. Two polynomial representations of experimental design. Journal of Statistical Theory and Practice, 2007. arXiv:0709.2997 1 (in press).

[6] Giovanni Pistone, Eva Riccomagno, and Maria Piera Rogantin. Algebraic statistics for the design of experiments. In Luc Pronzato and Antony A. Zigljavsky, editors, Search for Optimality in Design and Statistics: Algebraic and Dinamical System Methods, pages 95-129. Springer-Verlag, 2007.

[7] Giovanni Pistone, Eva Riccomagno, and Henry P. Wynn. Algebraic Statistics: Computational Commutative Algebra in Statistics. Chapman\&Hall, 2001.

[8] Giovanni Pistone and Maria-Piera Rogantin. Comparison of different definitions of regular fraction. Rapporti Interni 2007/2, Politecnico di Torino DIMAT, 2007.

[9] Giovanni Pistone and Maria Piera Rogantin. Indicator function and complex coding for mixed fractional factorial designs. Journal of Statistical Planning and Inference, 2007. Received 5 May 2005; revised 4 December 2006; accepted 8 February 2007. Available online 12 March 2007.

[10] R.L. Plackett and J.P. Burman. The design of optimum multifactorial experiments. Biometrika, 33:305-325, 1946.

[11] Kenny Q. Ye. Indicator function and its application in two-level factorial designs. The Annals of Statistics, 31(3):984-994, 2003.

DIMAT POLITECNICO DI TORINO

E-mail address: \{giovanni.pistone|roberto.fontana\}@polito.it 